\title{
A Coupling Process of Distillation with Vapor Permeation and Adsorption for Production of Fuel Ethanol: A Comparative Analysis on Energy Consumption
}

Jiacheng Wang¹, Jiaqi Zhang¹, Zhou Hong², Xuechao Gao ${ }^{1}$ *, Xuehong Gu1, *

1. State Key Laboratory of Materials-Oriented Chemical Engineering, College of Chemical Engineering, Nanjing Tech University, No. 30 Puzhu Road (S), Nanjing 211816, PR China

2. Nanjing Membrane Materials Industrial Technology Research Institute Co., Ltd, No. 1 Yuansi

Road, Pukou Economic Development Zone, Nanjing 211808, PR China

\section{A. Energy consumption analysis on the dissolved $\mathrm{CO}_{2}$ in feed}

The simplification for the feed ignoring the $\mathrm{CO}_{2}$ component was proposed based on the following calculation.

According to the solubility of $\mathrm{CO}_{2}$ in water under feed stream temperature $25{ }^{\circ} \mathrm{C}(1.449 \mathrm{~g} / \mathrm{L})$, the mass fraction of $\mathrm{CO}_{2}$ was set to be 0.0014 wt.\%. The energy consumption for distillation columns was calculated as shown in the following table.

\begin{tabular}{|l|l|l|l|}
\hline & feed with no CO2 & feed with CO2 & difference \\
\hline single-column process & $7965 \mathrm{~kW}$ & $7971 \mathrm{~kW}$ & $6 \mathrm{~kW}(0.075 \%)$ \\
\hline three-column process & $3748 \mathrm{~kW}$ & $3775 \mathrm{~kW}$ & $27 \mathrm{~kW}(0.72 \%)$ \\
\hline
\end{tabular}

Under the practical condition of the manufacturing processes, the dissolved $\mathrm{CO}_{2}$ could be controlled under $1 \mathrm{~g} / \mathrm{L} .{ }^{1}$ The energy cost by $\mathrm{CO}_{2}$ could be further declined.

1. Feng, S.; Lin, G. P., Development of dissolved carbon dioxide-driven-and-controlled repeated batch fermentation process for ethanol production. Can. J. Chem. Eng. 2019, 98, 2507-2515. 


\section{B. Stream Information}

The stream IDs in the four couplings were provided in Figure S1-S4, and the detailed information were given in Table S1-S4, respectively.

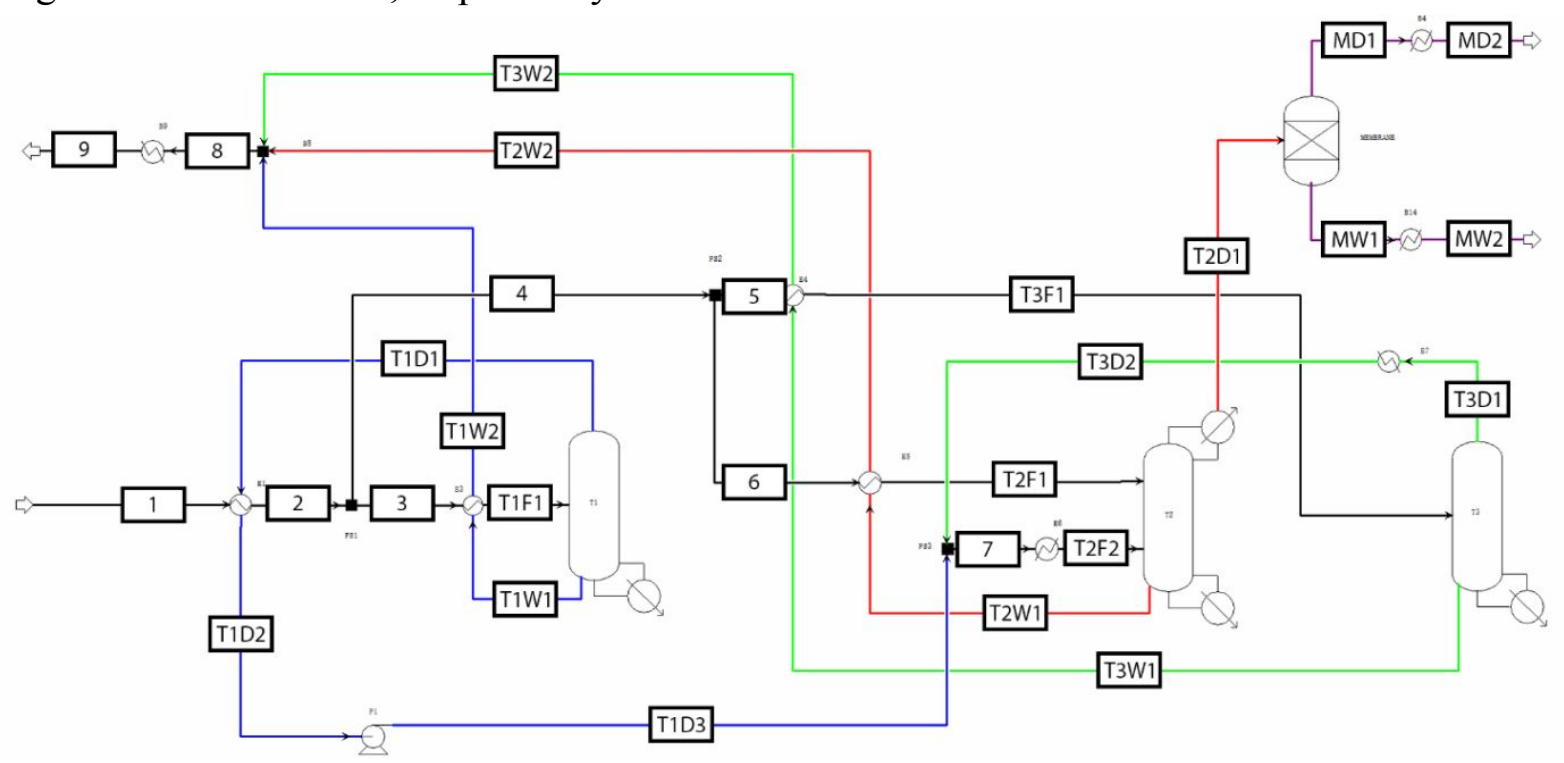

Figure S1 Stream ID for 3-DISTL VP process

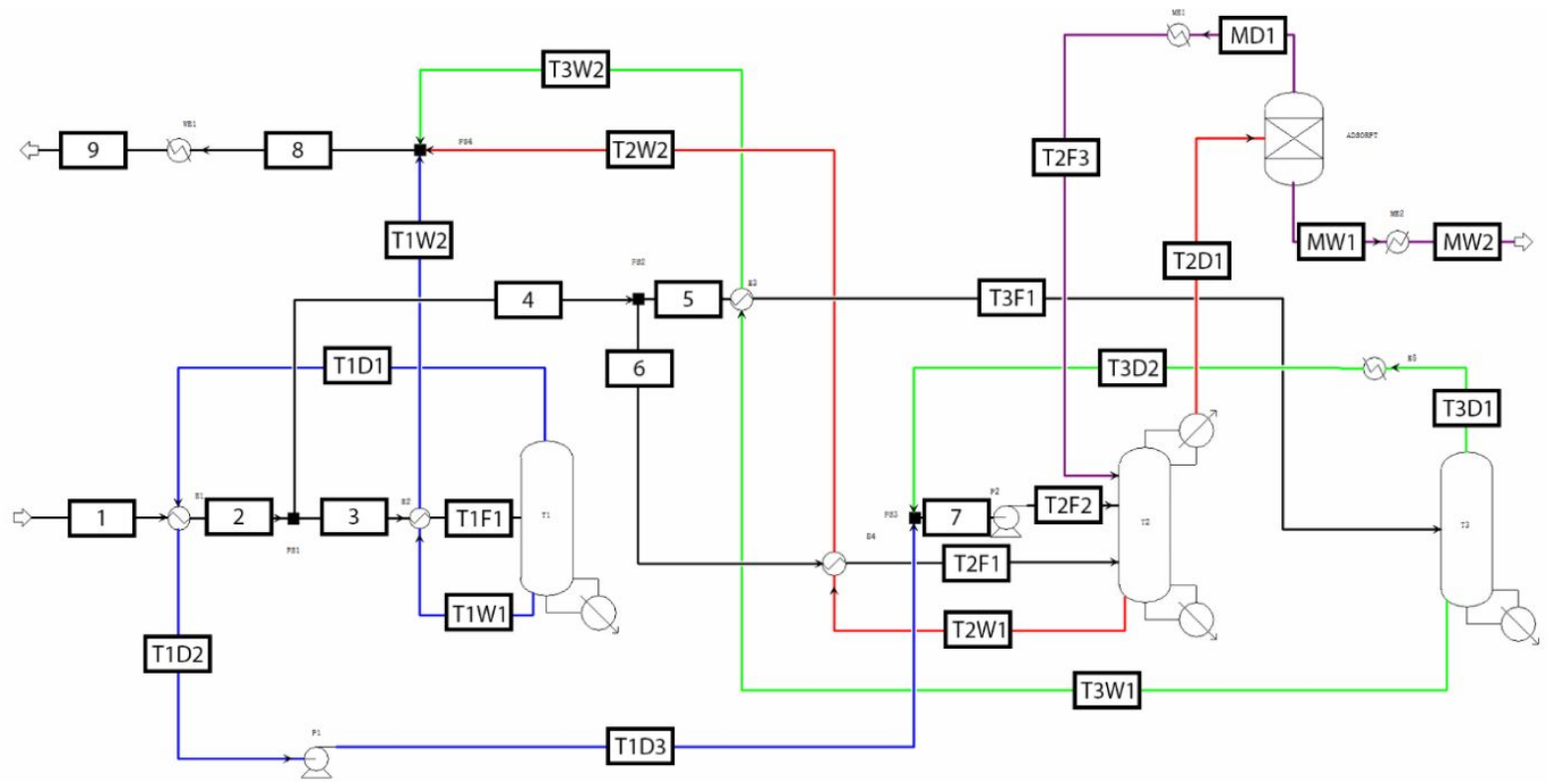

Figure S2 Stream ID for 3-DISTL AD process 


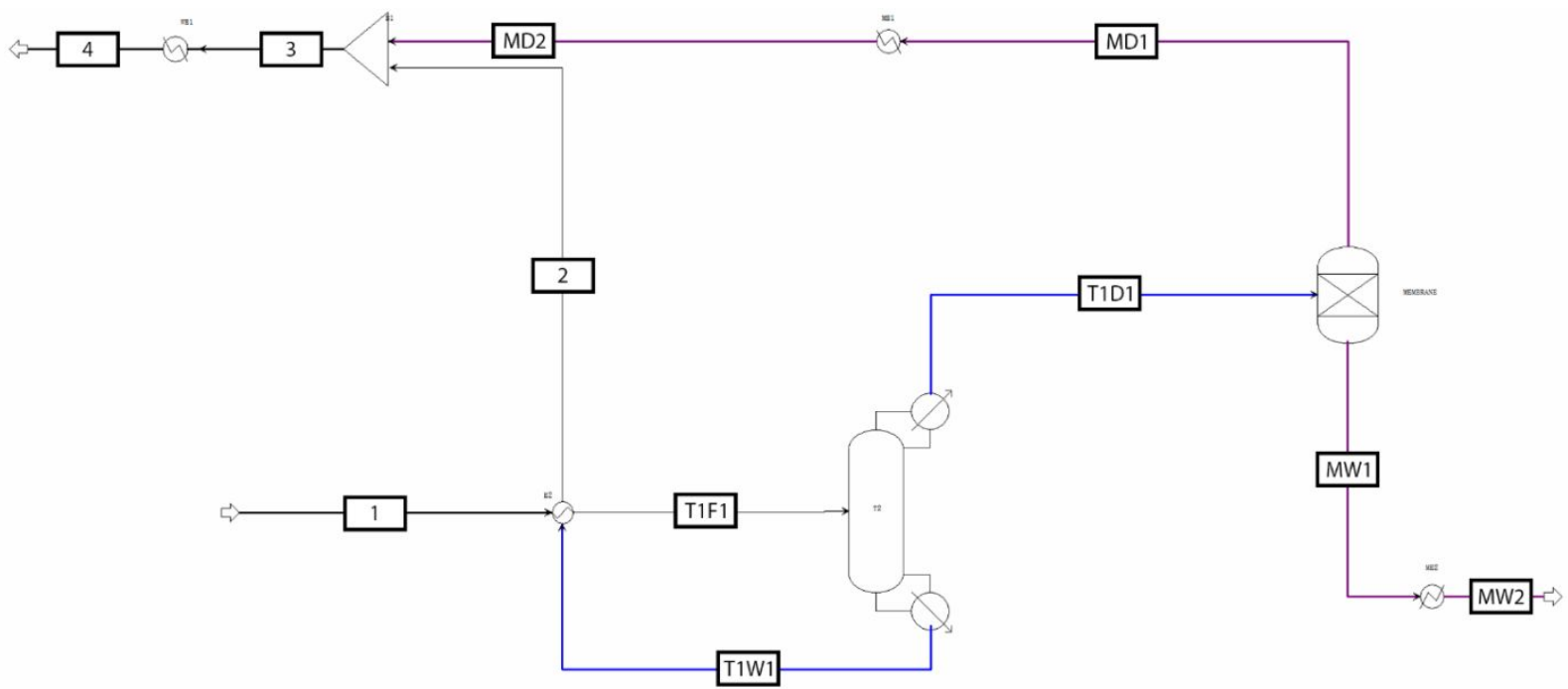

Figure S3 Stream ID for 1-DISTL VP process

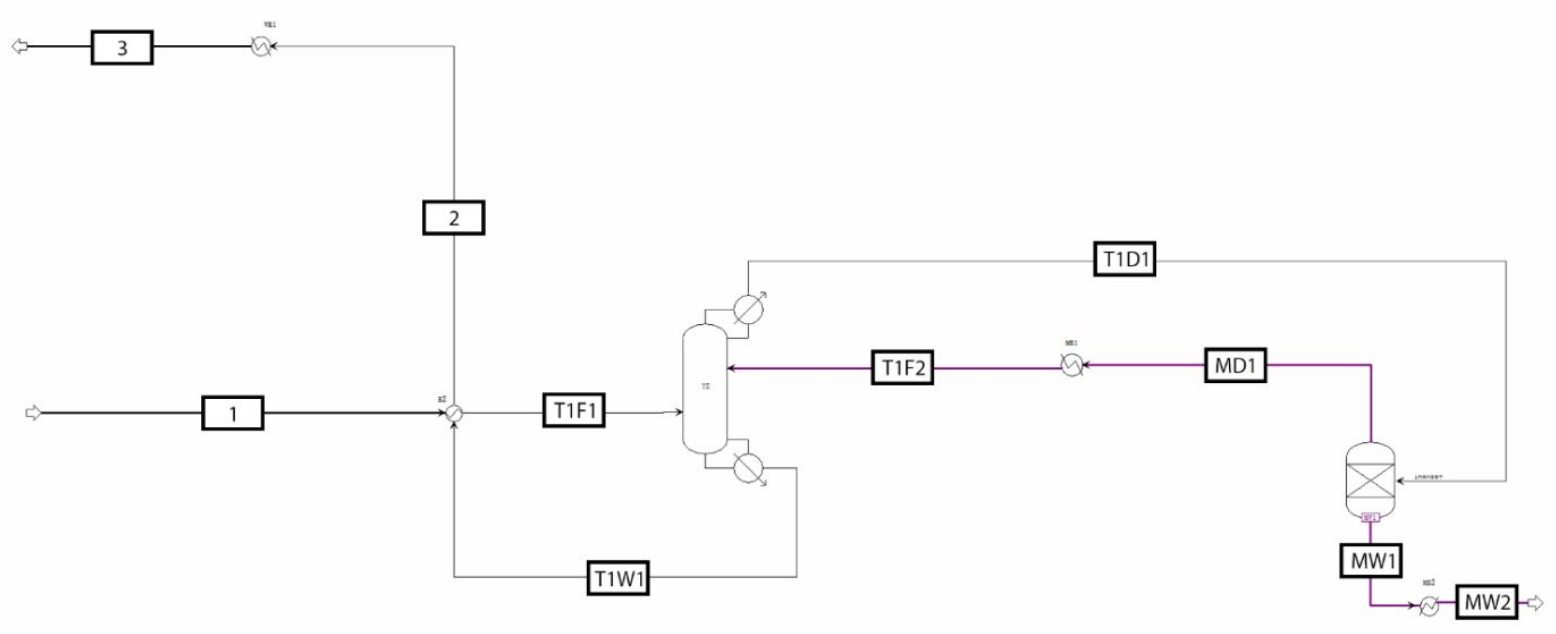

Figure S4 Stream ID for 1-DISTL AD process 
Table S1 Stream information for 3-DISTL VP process

\begin{tabular}{|c|c|c|c|c|c|c|c|}
\hline & Temperature (C) & Pressure (kPag) & Mass Flows (kg/hr) & ETHANOL (kg/hr) & WATER $(\mathrm{kg} / \mathrm{hr})$ & $\begin{array}{l}\text { ETHANOL (mass } \\
\text { frac) }\end{array}$ & $\begin{array}{l}\text { WATER (mass } \\
\text { frac) }\end{array}$ \\
\hline 1 & 25 & 600 & 83000 & 2490 & 80510 & 0.03 & 0.97 \\
\hline 2 & 37.18004 & 600 & 83000 & 2490 & 80510 & 0.03 & 0.97 \\
\hline 3 & 37.18004 & 600 & 17969.09 & 539.0726 & 17430.01 & 0.03 & 0.97 \\
\hline 4 & 37.18004 & 600 & 65030.91 & 1950.927 & 63079.99 & 0.03 & 0.97 \\
\hline 5 & 37.18004 & 600 & 22762.61 & 682.8782 & 22079.73 & 0.03 & 0.97 \\
\hline 6 & 37.18004 & 600 & 42268.31 & 1268.049 & 41000.26 & 0.03 & 0.97 \\
\hline 7 & 75.91108 & 0 & 4700.437 & 1221.939 & 3478.498 & 0.259963 & 0.740037 \\
\hline 8 & 64.46099 & -55 & 80091.48 & 2.214333 & 80089.27 & $2.76 \mathrm{E}-05$ & 0.999972 \\
\hline 9 & 40 & 200 & 80091.48 & 2.214333 & 80089.27 & $2.76 \mathrm{E}-05$ & 0.999972 \\
\hline T1F1 & 65 & 600 & 17969.09 & 539.0726 & 17430.01 & 0.03 & 0.97 \\
\hline T3F1 & 77.49453 & 600 & 22762.61 & 682.8782 & 22079.73 & 0.03 & 0.97 \\
\hline T2F1 & 125.3421 & 600 & 42268.31 & 1268.049 & 41000.26 & 0.03 & 0.97 \\
\hline $\mathrm{T} 2 \mathrm{~F} 2$ & 112.6376 & 600 & 4700.437 & 1221.939 & 3478.498 & 0.259963 & 0.740037 \\
\hline MD1 & 133.7078 & 200 & 409.6481 & 1.243893 & 408.4042 & 0.003036 & 0.996964 \\
\hline MD2 & 99.6386 & 0 & 409.6481 & 1.243893 & 408.4042 & 0.003036 & 0.996964 \\
\hline MW1 & 125.3585 & 400 & 2500.043 & 2486.542 & 13.50097 & 0.9946 & 0.0054 \\
\hline MW2 & 131.6863 & 500 & 2500.043 & 2486.542 & 13.50097 & 0.9946 & 0.0054 \\
\hline T1D1 & 73.84488 & -60 & 2073.632 & 539.0614 & 1534.571 & 0.25996 & 0.74004 \\
\hline T1D2 & 63.77722 & -60 & 2073.632 & 539.0614 & 1534.571 & 0.25996 & 0.74004 \\
\hline T1D3 & 63.82215 & 0 & 2073.632 & 539.0614 & 1534.571 & 0.25996 & 0.74004 \\
\hline T1W1 & 79.4504 & -55 & 15895.45 & 0.011171 & 15895.44 & 7.03E-07 & 0.999999 \\
\hline T1W2 & 48.71726 & -55 & 15895.45 & 0.011171 & 15895.44 & 7.03E-07 & 0.999999 \\
\hline T2D1 & 125.6385 & 400 & 2909.691 & 2487.786 & 421.9052 & 0.855 & 0.145 \\
\hline $\mathrm{T} 2 \mathrm{~W} 1$ & 152.7542 & 410 & 44060.23 & 2.203012 & 44058.03 & 0.00005 & 0.99995 \\
\hline T2W2 & 72 & 410 & 44060.23 & 2.203012 & 44058.03 & 0.00005 & 0.99995 \\
\hline T3D1 & 96.73604 & 0 & 2626.805 & 682.878 & 1943.927 & 0.259965 & 0.740035 \\
\hline T3D2 & 85.29239 & 0 & 2626.805 & 682.878 & 1943.927 & 0.259965 & 0.740035 \\
\hline T3W1 & 103.9359 & 15 & 20135.8 & 0.000151 & 20135.8 & 7.48E-09 & 1 \\
\hline T3W2 & 60 & 15 & 20135.8 & 0.000151 & 20135.8 & 7.48E-09 & 1 \\
\hline
\end{tabular}


Table S2 Stream information for 3-DISTL AD process

\begin{tabular}{|c|c|c|c|c|c|c|c|}
\hline & Temperature (C) & Pressure (kPag) & Mass Flows $(\mathrm{kg} / \mathrm{hr})$ & ETHANOL (kg/hr) & WATER (kg/hr) & $\begin{array}{l}\text { ETHANOL (mass } \\
\text { frac) }\end{array}$ & $\begin{array}{l}\text { WATER (mass } \\
\text { frac) }\end{array}$ \\
\hline 1 & 25 & 600 & 83000 & 2490 & 80510 & 0.03 & 0.97 \\
\hline 2 & 38.45839 & 600 & 83000 & 2490 & 80510 & 0.03 & 0.97 \\
\hline 3 & 38.45839 & 600 & 19867.57 & 596.0272 & 19271.55 & 0.03 & 0.97 \\
\hline 4 & 38.45839 & 600 & 63132.43 & 1893.973 & 61238.45 & 0.03 & 0.97 \\
\hline 5 & 38.45839 & 600 & 24620.4 & 738.6121 & 23881.79 & 0.03 & 0.97 \\
\hline 6 & 38.45839 & 600 & 38512.02 & 1155.361 & 37356.66 & 0.03 & 0.97 \\
\hline 7 & 75.79519 & 0 & 5133.78 & 1334.574 & 3799.206 & 0.259959 & 0.740041 \\
\hline 8 & 52.77326 & -55 & 80499.58 & 2.089647 & 80497.49 & $2.6 \mathrm{E}-05$ & 0.999974 \\
\hline 9 & 40 & 200 & 80499.58 & 2.089647 & 80497.49 & $2.6 \mathrm{E}-05$ & 0.999974 \\
\hline MD1 & 115.5839 & 200 & 2258.417 & 1580.892 & 677.5251 & 0.7 & 0.3 \\
\hline MW1 & 125.3632 & 400 & 2500.39 & 2487.888 & 12.50191 & 0.995 & 0.005 \\
\hline MW2 & 125.3547 & 400 & 2500.39 & 2487.888 & 12.50191 & 0.995 & 0.005 \\
\hline $\mathrm{T} 2 \mathrm{~F} 3$ & 138.5919 & 600 & 2258.432 & 1580.903 & 677.5297 & 0.7 & 0.3 \\
\hline T1D1 & 73.84494 & -60 & 2292.585 & 595.9704 & 1696.615 & 0.259956 & 0.740044 \\
\hline T1D2 & 63.77732 & -60 & 2292.585 & 595.9704 & 1696.615 & 0.259956 & 0.740044 \\
\hline T1D3 & 63.82225 & 0 & 2292.585 & 595.9704 & 1696.615 & 0.259956 & 0.740044 \\
\hline T1F1 & 66.46708 & 600 & 19867.57 & 596.0272 & 19271.55 & 0.03 & 0.97 \\
\hline $\mathrm{T} 1 \mathrm{~W} 1$ & 79.45034 & -55 & 17573.84 & 0.022314 & 17573.82 & $1.27 \mathrm{E}-06$ & 0.999999 \\
\hline T1W2 & 48.45839 & -55 & 17573.84 & 0.022314 & 17573.82 & $1.27 \mathrm{E}-06$ & 0.999999 \\
\hline $\mathrm{T} 2 \mathrm{D} 1$ & 118.3149 & 300 & 4758.807 & 4068.78 & 690.027 & 0.855 & 0.145 \\
\hline T2F1 & 134.6902 & 600 & 38512.02 & 1155.361 & \begin{tabular}{|l}
37356.66 \\
\end{tabular} & 0.03 & 0.97 \\
\hline $\mathrm{T} 2 \mathrm{~F} 2$ & 76.17909 & 600 & 5133.78 & 1334.574 & 3799.206 & 0.259959 & 0.740041 \\
\hline $\mathrm{T} 2 \mathrm{~W} 1$ & 144.6902 & 310 & 41146.72 & 2.057336 & 41144.66 & 0.00005 & 0.99995 \\
\hline $\mathrm{T} 2 \mathrm{~W} 2$ & 56.80143 & 310 & 41146.72 & 2.057336 & 41144.66 & 0.00005 & 0.99995 \\
\hline T3D1 & 96.73608 & 0 & 2841.194 & 738.6035 & 2102.591 & 0.259962 & 0.740038 \\
\hline T3D2 & 85.29247 & 0 & 2841.194 & 738.6035 & 2102.591 & 0.259962 & 0.740038 \\
\hline T3F1 & 88.67789 & 600 & 24620.4 & 738.6121 & 23881.79 & 0.03 & 0.97 \\
\hline T3W1 & 103.9359 & 15 & 21779.02 & 0.009997 & 21779.01 & $4.59 \mathrm{E}-07$ & 1 \\
\hline
\end{tabular}




\begin{tabular}{|c|c|c|c|c|c|c|c|}
\hline T3W2 & 48.45839 & 15 & 21779.02 & 0.009997 & 21779.01 & 4.59E-07 & 1 \\
\hline
\end{tabular}

Table S3 Stream information for 1-DISTL VP process

\begin{tabular}{|c|c|c|c|c|c|c|c|}
\hline & Temperature $(\mathrm{C})$ & Pressure (kPag) & Mass Flows $(\mathrm{kg} / \mathrm{hr})$ & ETHANOL (kg/hr) & WATER (kg/hr) & $\begin{array}{l}\text { ETHANOL (mass } \\
\text { frac) }\end{array}$ & $\begin{array}{l}\text { WATER (mass } \\
\text { frac) }\end{array}$ \\
\hline 1 & 25 & 600 & 83000 & 2490 & 80510 & 0.03 & 0.97 \\
\hline 2 & 35 & 410 & 80092.4 & 4.00462 & 80088.4 & 0.00005 & 0.99995 \\
\hline 3 & 35.56978 & 200 & 80505.67 & 8.137239 & 80497.53 & 0.000101 & 0.999899 \\
\hline 4 & 40 & 200 & 80505.67 & 8.137239 & 80497.53 & 0.000101 & 0.999899 \\
\hline MD1 & 133.6198 & 200 & 413.2624 & 4.132619 & 409.1297 & 0.01 & 0.99 \\
\hline MW1 & 125.3632 & 400 & 2494.335 & 2481.863 & 12.47182 & 0.995 & 0.005 \\
\hline MW2 & 125.3547 & 400 & 2494.335 & 2481.863 & 12.47182 & 0.995 & 0.005 \\
\hline $\mathrm{T} 1 \mathrm{~F} 1$ & 141.0067 & 600 & 83000 & 2490 & 80510 & 0.03 & 0.97 \\
\hline MD2 & 132.2666 & 200 & 413.2624 & 4.132619 & 409.1297 & 0.01 & 0.99 \\
\hline T1D3 & 125.6385 & 400 & 2907.597 & 2485.995 & 421.6016 & 0.855 & 0.145 \\
\hline T1W1 & 152.7542 & 410 & 80092.4 & 4.00462 & 80088.4 & 0.00005 & 0.99995 \\
\hline
\end{tabular}

\begin{tabular}{|c|c|c|c|c|c|c|c|}
\hline & Temperature (C) & Pressure (kPag) & Mass Flows $(\mathrm{kg} / \mathrm{hr})$ & ETHANOL (kg/hr) & WATER (kg/hr) & $\begin{array}{l}\text { ETHANOL (mass } \\
\text { frac) }\end{array}$ & $\begin{array}{l}\text { WATER (mass } \\
\text { frac) }\end{array}$ \\
\hline 1 & 25 & 600 & 83000 & 2490 & 80510 & 0.03 & 0.97 \\
\hline 2 & 35 & 310 & 80501.53 & 4.025077 & 80497.51 & 0.00005 & 0.99995 \\
\hline 3 & 40 & 200 & 80501.53 & 4.025077 & 80497.51 & 0.00005 & 0.99995 \\
\hline MD1 & 115.5839 & 200 & 2256.68 & 1579.676 & 677.0039 & 0.7 & 0.3 \\
\hline MW1 & 125.3632 & 400 & 2498.467 & 2485.974 & 12.49229 & 0.995 & 0.005 \\
\hline MW2 & 125.3547 & 400 & 2498.467 & 2485.974 & 12.49229 & 0.995 & 0.005 \\
\hline T1F1 & 133.5755 & 600 & 83000 & 2490 & 80510 & 0.03 & 0.97 \\
\hline T1F2 & 138.5919 & 600 & 2256.679 & 1579.675 & 677.0036 & 0.7 & 0.3 \\
\hline T1D1 & 118.3149 & 300 & 4755.146 & 4065.65 & 689.4962 & 0.855 & 0.145 \\
\hline T1W1 & 144.6902 & 310 & 80501.53 & 4.025077 & 80497.51 & 0.00005 & 0.99995 \\
\hline
\end{tabular}




\section{Modeling Methods \\ C1. Multi-effect distillation}

The multi-effect distillation was fulfilled by the two pairs of energy matching calculations between the higher-pressure distillation column and the lower-pressure distillation column, through setting the required design specifications, which can be achieved by iterations of the two split ratios for the feed flow in the process. A detailed instruction is given as following steps in Aspen Plus.

Step 1 Prework includes finishing the settings of the distillation process with 3 columns, named T1 (LP column), T2 (HP column), T3 (MP column) and 2 splitters, named FS1 (splitting feed flow for T1), FS2 (splitting feed flow for T2 and T3).

Step 2 Under the Flowsheeting Options menu, create 2 design specs, named DS-1 and DS-2.

$\underline{\text { Step } 3}$ To match the energy cost between T1 and T3, under DS-1, create two variables, named QC3 and QR1. QC3 is a block variable for the cooling duty of T3. QR1 is a block variable for the heating duty of T1.

Step 4 Set the design specification expression to be $Q C 3+Q R 1=0$ with tolerance 0.1 . Set the manipulated variable for the fraction in FS1 in the range from 0.1 to 0.9 .

Step 5 To match the energy cost between T3 and T2, under DS-2, create two variables, named QC2 and QR3. QC2 is a block variable for the cooling duty of T2. QR3 is a block variable for the heating duty of T3.

$\underline{\text { Step } 6}$ Set the design specification expression to be QC2+QR3=0 with tolerance 0.1. Set the manipulated variable for the fraction in FS2 in the range from 0.1 to 0.9 .

$\underline{\text { Step } 7}$ Run the simulation and check the results for the energy matching.

C2. Vapor permeation model

The model parameters and model settings could be referred as following.

Variables in each flow of a single cell as shown in Figure 2 (retentate side in/out, permeate side in/out and permeate flow)

$\begin{array}{lll}\text { TReti } & \text { as } & \text { temperature; } \\ \text { TVapi } & \text { as } & \text { temperature; } \\ \text { TReto } & \text { as } & \text { temperature; } \\ \text { TVapo } & \text { as } & \text { temperature; } \\ \text { TPerm } & \text { as } & \text { temperature; } \\ \text { PRet } & \text { as } & \text { pressure; } \\ \text { PVap } & \text { as } & \text { pressure; } \\ \text { ZReti(ComponentList) } & \text { as } & \text { massfraction; } \\ \text { ZVapi(ComponentList) } & \text { as } & \text { massfraction; } \\ \text { ZReto(ComponentList) } & \text { as } & \text { massfraction; } \\ \text { ZVapo(ComponentList) } & \text { as } & \text { massfraction; } \\ \text { ZPerm(ComponentList) } & \text { as } & \text { massfraction; } \\ \text { FReti } & \text { as } & \text { flow_mass; } \\ \text { FVapi } & \text { as } & \text { flow_mass; } \\ \text { FReto } & \text { as } & \text { flow_mass; } \\ \text { FVapo } & \text { as } & \text { flow_mass; } \\ \text { FPerm } & \text { as } & \text { flow_mass; }\end{array}$


Coefficient for permeability

$\begin{array}{lll}\mathrm{k} & \text { as } & \text { notype; } \\ \mathrm{E} & \text { as } & \text { notype; }\end{array}$

Mass balance in a single cell

For comp in ComponentList Do

FReti*ZReti (comp) $=$ FReto*ZReto $($ comp $)+$ FPerm*ZPerm (comp);

FVapi*ZVapi(comp) + FPerm*ZPerm(comp) = FVapo*ZVapo(comp);

Endfor

sigma $($ ZReto $)=1$;

$\operatorname{sigma}(Z \mathrm{Z}$ apo $)=1$

$\operatorname{sigma}($ ZPerm $)=1$;

TPerm = TReti;

TReto = TReti;

TVapo = TReti;

Membrane separation process for water component

Fperm*Zperm=k*exp(-E/8.314*Tperm)*(PRet*ZReto-PVap*ZVapo);

Set input/output ports and transfer the values

$\begin{array}{llll}\text { Rinlet } & \text { as } & \text { input } & \text { main; } \\ \text { Vinlet } & \text { as } & \text { input } & \text { main; } \\ \text { Routlet } & \text { as } & \text { output } & \text { main; } \\ \text { Voutlet } & \text { as } & \text { output } & \text { main; }\end{array}$

Variables for multiple cells

$\begin{array}{llll}\text { Ncells } & \text { as } & \text { IntegerParameter } & \text { (value:10); } \\ \text { stage([1:Ncells }]) & \text { as } & \text { single_cell; } \\ \text { A } & \text { as } & \text { Area; }\end{array}$

stage. ACell $=\mathrm{A} / \mathrm{NCells}$;

Transfer the values and connect each cell

For $\mathrm{j}$ in [1:Ncells] do

stage $(\mathrm{j}) \cdot \mathrm{k}=\mathrm{k}$;

stage $(\mathrm{j}) \cdot \mathrm{E}=\mathrm{E}$;

Endfor

For $\mathrm{j}$ in $[1:$ Ncells- 1$]$ do connect stage $(\mathrm{j}) \cdot$ Routlet and stage $(\mathrm{j}+1)$.Rinlet; connect stage $(\mathrm{j})$.Voutlet and stage $(\mathrm{j}+1)$.Vinlet;

Endfor 


\section{C3. Adsorption model}

The adsorption model was fulfilled by the SEP module, where the recovery rate for each component was determined according to the industrial process in factories, with a recycling sweep flow was simulated, being induced back into the HP column.

The simulation files could be obtained by contacting the corresponding authors. 